This item was submitted to Loughborough's Research Repository by the author.

Items in Figshare are protected by copyright, with all rights reserved, unless otherwise indicated.

\title{
A relational oriented approach to system of systems assessment of alternatives for data link interoperability
}

PLEASE CITE THE PUBLISHED VERSION

http://dx.doi.org/10.1109/JSYST.2013.2254215

PUBLISHER

(C) IEEE

VERSION

AM (Accepted Manuscript)

LICENCE

CC BY-NC-ND 4.0

\section{REPOSITORY RECORD}

Dickerson, Charles E.. 2019. "A Relational Oriented Approach to System of Systems Assessment of Alternatives for Data Link Interoperability”. figshare. https://hdl.handle.net/2134/13081. 
This item was submitted to Loughborough's Institutional Repository (https://dspace.lboro.ac.uk/) by the author and is made available under the following Creative Commons Licence conditions.

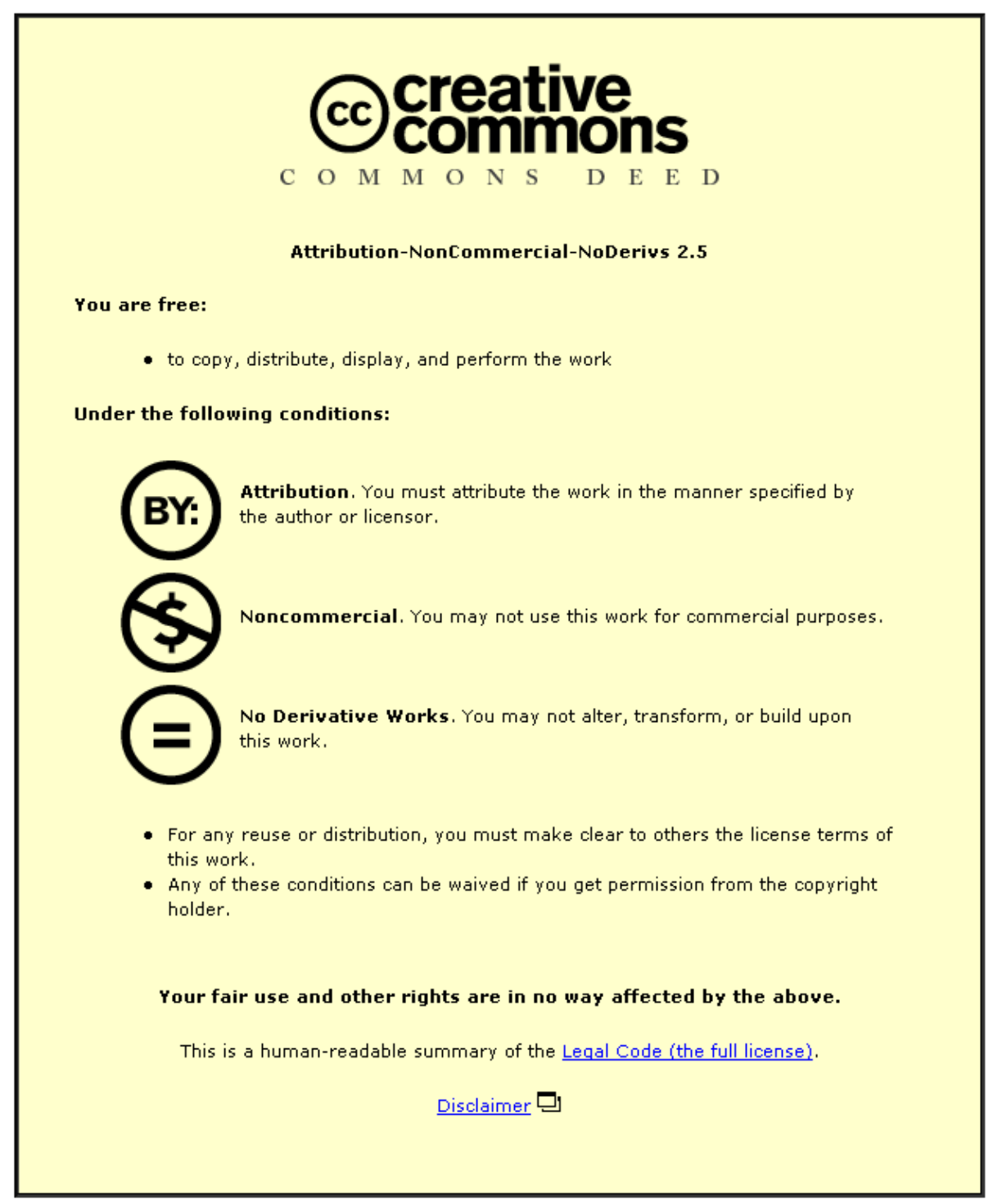

For the full text of this licence, please go to: http://creativecommons.org/licenses/by-nc-nd/2.5/ 


\title{
A Relational Oriented Approach to System of Systems Assessment of Alternatives for Data Link Interoperability
}

\author{
Charles E. Dickerson, Member, IEEE
}

\begin{abstract}
Relational Oriented Systems Engineering (ROSE) is applied to a large scale system of systems tactical data link interoperability problem. A model driven framework structure developed using the ROSE methodology is employed to prescribe a repeatable approach for determining viable candidate solutions that completes and makes rigorous a previous capability based exploratory analysis performed by the Office of the Chief Engineer of the U.S. Navy. This novel and efficient approach to a long standing problem concentrates on the relationships between models to provide a framework and factorization of a system of systems architecture for portfolio selection and evaluation. The approach is demonstrated in a simplified but end-to-end case study derived from the original data link interoperability analysis. The abstract approach employed can be applied to a much wider class of problems than data link interoperability.
\end{abstract}

Index Terms - System of systems, architecture, systems engineering, model, transformation, object orientation, frame, framework, relational orientation, relational structure, interoperability, interface, Link 16, tactical data link, capability based acquisition, assessment of alternatives, case study.

\section{INTRODUCTION}

$\mathrm{T}^{\mathrm{s}}$ ACTICAL DATA LINKS (TDLs) are deployed links that support communication and command and control (C3) of forces operating in theater. Link 16 is a TDL that has been widely deployed across US Joint Forces as well as across international coalition forces to enable tactical data to be exchanged securely. It is a critical technology for the interoperability of the systems of systems (SoS) within these forces. Link 16 is a high-speed jam resistant network using Time Division Multiple Access that supports exchange of image and text data in near-real time, with the capability of providing digital voice channels through multiple, simultaneous communication paths [1]. It should be noted that in recent years Link 16 has been absorbed into what is now called Link 22.

\section{A. Assessment of Alternatives (AoA)}

Interoperability assessments are a significant challenge across a complex enterprise such as the U.S. Department of Defense

Manuscript received $30^{\text {th }}$ November 2011; revised $1^{\text {st }}$ March 2013. This work was supported in part by the Royal Academy of Engineering and the Engineering and Physical Sciences Research Council, UK.

C.E. Dickerson is with the School of Electronic, Electrical and Systems Engineering, Loughborough University, Leicestershire, LE11 3TU, UK Digital Object Identifier....
(DoD). For example, the Joint Interoperability Test Command in an annual status report [2] supported 244 test activities involving 154 DoD systems, of which Link 16 was only one. But Assessment of Alternatives (AoA) for SoS interoperability improvements to the TDLs is an even greater challenge because data links enable the operational capability of the SoS. Therefore the tradeoffs have a complexity of much greater scope than just deciding between the $\mathrm{C} 3$ alternatives and performing interoperability assessments.

\section{B. Problem Statement and Background}

In the year 2003, the U.S. Navy Center for Tactical Systems Interoperability (NCTSI) conducted a trade study on Link 16 issues associated with deployed systems that could be addressed by implementing one or more of 137 Interface Change Proposals (ICPs) submitted for Link 16 improvement by various Navy System Commands and Joint Working Groups. An ICP could specify change of information needs and propose physical and/or software changes to the interfaces. Each ICP was intended to address a given operational requirement and each included an estimated cost to implement the proposed change.

Operating under a constrained budget, NCTSI needed to select a combination of the ICPs that would provide the most improvement of fleet mission capabilities. This was in the early years of Capability Based Acquisition (CBA) in the U.S. DoD. One of the challenges was to quantify what was meant by 'improvement of capabilities'. Individual systems or even a combination of systems meeting their allocated requirements was not sufficient to ensure that the fleet could meet its mission objectives. Details of various approaches to and challenges of CBA can be found in Sections IIB and IV.

The Deputy Assistant Secretary of the Navy (DASN), for Integrated Weapon Systems (IWS), suggested that the Office of the Chief Engineer of the Navy, working with the Space Warfare Systems Command (SPAWAR) and NTCSI, might offer some new insights as to how best to approach this problem using the then recent advances in CBA.

The capability based approach to the AoA was successful, giving new insight as to how the tradeoffs might better be performed and making recommendations as to which ICPs to acquire. However, although successful and documented in an open source by the author in [3], the original work was never successfully formalized into a repeatable analysis approach. 
To say that an approach is repeatable in this paper means that it is sufficiently prescribed that the same analysis could be performed outside of the original team and reproduce the original solution.

\section{Specification of Relational Orientation}

In Section III, relational orientation will be used to prescribe a repeatable methodology for the AoA analysis. Relational oriented systems engineering (ROSE) as introduced in [4] is a general systems methodology similar to [5] and employs a principle of model specification and relational transformation for the purpose of system description, analysis, and design. From the relational viewpoint, the specification of a model associated with a system is the specification of:

- Entities associated with the system

- Sentences (declarations) about the entities

- Modeling elements to instantiate the sentences

- A semantic structure on the modeling elements

- Interpretations of the sentences into the semantic structure

Entities are abstractions that admit logical or physical existence. The entities of the system can include attributes, classes, and components of the system. There can also be entities associated with the system which are not part of it, e.g. the environment. The sentences are the basis for system specification. The system model is valid when the interpretation of each sentence is true within the semantic structure of the model. The validation process is facilitated by two types of semantic structures: relational structures (i.e. a collection of mathematical relations) and graphical models (e.g. class diagrams). An architectural domain relates to a type of knowledge about the system or to one or more system components. System architecture has been conceptualized through relationships by ISO [6].

Semantic structure is a concept which seeks to formalize the intended meaning of natural language through some type of organization [7]. Relational orientation is primarily concerned with two types of semantic structures: relational structures and graphical models. These will be referred to as relational frames and specified as ordered pairs $(M, \mathrm{R})$. The modeling elements are specified by $M$ and the semantic structure is specified by $\mathrm{R}$. When $M$ is a collection of mathematical objects, such as numbers or sets, the structure of the frame will be specified by a relational structure $\mathrm{R}=\left\{\mathrm{R}_{\alpha}\right\}$ on $M$ to capture semantic knowledge. A graphical model is a collection of vertices and edges for encoding the semantic knowledge captured by the sentences. The modeling elements in this case are the vertices. The edges, which represent relations between vertices, are represented as pairs of vertices. Thus each $R_{\alpha}$ in the frame of a graphical model is a pair of vertices.

Relational frames provide a static structure for organizing knowledge about the system using predefined internal relations specified by the model that reflect the relational structure of the semantic knowledge captured by the sentences and their interpretation into the structure. This extends the concept of object oriented frames used in software engineering as in [8] which is primarily concerned with the classes and objects of software architecture, their responsibilities and collaborations, and the threads of control.

Model elements are related by belonging to: (i) n-ary mathematical relations, (ii) hierarchical decomposition, or (iii) association with elements of another model by transformation. The special case $n=1$ for $n$-ary relations is a unary relation, i.e. a defined subset of elements. The first two types of relation correspond to the internal structure of the model. The associated relational frames will be referred to simply as frames. The third type of relation can be an association external to the model, although hierarchical decomposition into sub-models can also admit transformations. The frame will be referred to as a transformational frame and denoted as $\underline{Q}=(M, N ; \mathrm{Q})$ where $M$ and $N$ are the elements of two models and $\mathrm{Q}$ is an association between the structures of the frames.

A relational transformation is specified as an association between the elements of two models of a system that induces a mapping between the relationships expressed in the models and preserves the structure of these relationships. For example, a relational transformation of a graph can be defined by a multi-valued association of the vertices as in Fig. 1 that preserves the relationships of the edges to the vertices.
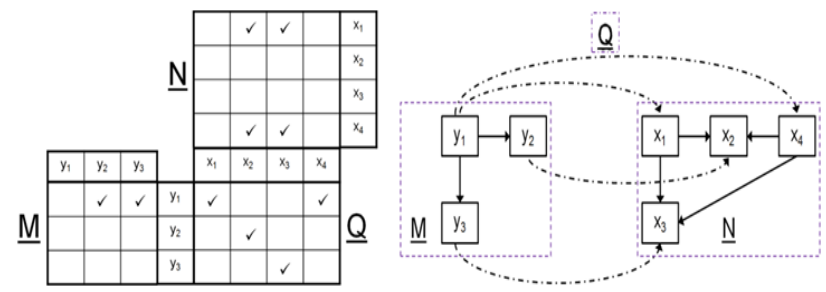

Fig. 1. Example of Relational Transformation Framework

When two models of a system are related by a relational transformation, the collective three frames will be referred to as a framework. In relational orientation, systems are modeled using multiple frameworks, which represent the various knowledge domains and components of the system. Frameworks are integrated into a framework structure by sharing common frames or by transformations between frames.

The specification of frames for the models and transformational frames between the models is complete when they form a framework structure that is adequate for system specification, analysis and design. This resultant framework structure provides a metamodel of the system, i.e. an abstraction used for specifying the models of the system. This will provide the basis for the Link 16 AoA analysis.

\section{Meeting the AoA Challenge for SoS}

The approach used in the original NCTSI spreadsheet analysis was requirements based. Operational requirements 
were allocated to systems and the Link 16 ICPs were assessed by how much improvement the systems, allocated the ICPs, exhibited towards fulfilling the requirements. The technical description and operational sequences of activities and systems that accomplish the execution of the mission requirements are known as Operational Mission Threads (OMT) [9]. The approach used by the Office of the Chief Engineer was capability based. The ICPs were assessed by how much improvement in capability the allocated SoS exhibited, as measured by increased numbers of mission threads made interoperable. Neither offered a repeatable analysis approach.

The approach proposed in Section III of this paper is model driven and relationally oriented. ROSE is used to implement a capability based approach with provisions for a requirement based approach. Systems in the SoS achieve operational capabilities not just through individual actions but also through collective actions and interoperations as organized by allocations to a specified operational sequence associated with one or more mission objectives. The models specified using relational orientation will be organized into a metamodel defined by an integrated framework structure.

\section{THE TWO LEGACY ANALYSIS APPROACHES}

This section provides an overview of the requirements based approach used by NCTSI and the capabilities based approach used by the Office of the Chief Engineer.

\section{A. Requirements Based Approach}

The initial focus of the NCTSI tradeoff study had been to use a spreadsheet like the one in Fig. 2 which illustrates how the trades were visualized. Details of the information in the spreadsheet can be found in tables provided in this section and in Section III. Each row in the spreadsheet corresponded to an ICP which was identified by a number and a title called out in one of the columns and was also associated with the operational requirement the ICP was intended to support. The columns in the color-coded portion of the spreadsheet corresponded to the various systems to be improved through implementing an ICP. Each of these systems was supported by a Link 16 node. The scheme for the color-coded cells indicated the level of improvement expected from implementing the given ICP on the Link 16 node for the system. Thus, the NCTSI tradeoff analysis approach was straightforward and easy to visualize. The goal was to increase the number green cells through the implementation of a combination of the ICPs.

A large number of ICPs were based on the application of one or more so-called J-series messages. These are standardized messages that format specified data for use in the Link 16 schema. Of the 137 ICPs submitted, 76 were mapped to applicable J-series messages using the Operating Standard 516.2 and the ICP descriptions. The left most column in the spreadsheet indicates associations of J-series messages with the 76 ICPs. These messages became the subject of the analysis conducted by the Office of the Chief Engineer.

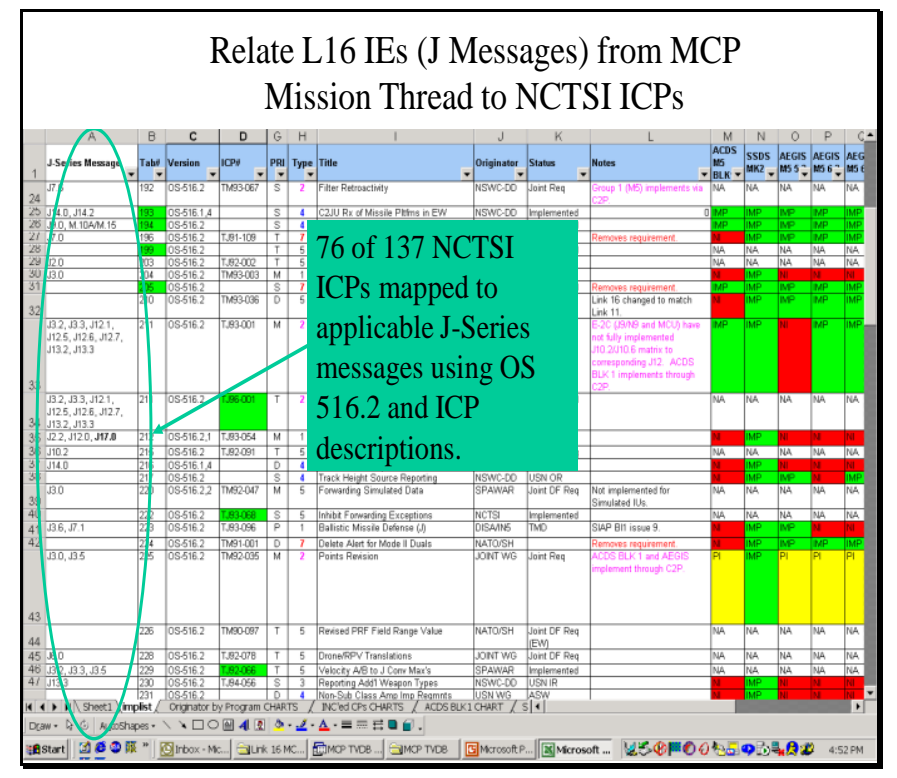

Fig. 2. NCTSI Link 16 ICP interoperability assessment spreadsheet analysis

\section{B. Capability Based Approach}

Before DASN IWS suggested that the NCTSI trade study might benefit from collaboration with the Office of the Chief Engineer, Mission Capabilities Packages (MCPs) had recently been introduced into CBA [10]. The Chief Engineer had just finished work with the Chief of Naval Operations to develop over a dozen MCPs that supported the tradeoff analyses for the Program Objective Memorandum for the 2004 fiscal year (POM04). The DoD Architecture Framework (DoDAF) [11] was used to structure information collected for the tradeoffs.

The most stressing missions for Link 16 were found in the Strike MCP. This is not surprising because of the increasing attention on time sensitive targeting at that time [3]. It was therefore decided to use the Strike MCP as the baseline for the Link 16 capability tradeoffs. In support of the POM04 Strike MCP, 39 mission threads had been populated and analyzed using the POM04 guidelines.

Each of the 39 mission threads represented an instance of operational capability for the strike mission. An analysis team was formed by the members of SPAWAR and the Office of the Chief Engineer who had worked on the Strike MCP for POM04. Using multiple databases and the NCTSI spreadsheet, the team employed a combination of automation and collaboration in a visualization environment to review and update the detailed DoDAF Systems View 6 (SV-6) compiled for the Strike MCP. The data collected for this view used in POM04 comprised 2,857 lines of connectivity information which were accessed as part of the interoperability analysis.

In the Link 16 analysis performed by the Office of the Chief Engineer, as with the POM04 Strike MCP capability tradeoff analyses, the interoperability assessments were based on an end-to-end analysis of each of the 39 mission threads using the 
SV-6 information on functionality, connectivity, and compliance with various standards (e.g. the United States Message Text Format (USMTF) [12]).

\section{1) Mapping Interoperability to Mission Capability:} Operational sequences where used to model mission capabilities. A simplified version of the operational sequence for Operational Mission Thread (OMT) \#14 from Tactical Situation-4 (TACSIT-4) of the Western Pacific (WESTPAC) scenario is displayed in Fig. 3 using the standard Find, Fix, Track, Target, Engage, and Assess model $\left(\mathrm{F}^{2} \mathrm{~T}^{2} \mathrm{EA}\right)$ and other requisite activities. The Fix (Fx) and Assess (A) activities are instantiated multiple times through the sequence and therefore are indexed to indicate the order of instantiation. Each numbered 'firecracker' is an interoperability gap traceable to Link 16 when the operational sequence was instantiated in TACSIT-4. This type of illustration of the gaps displays where the ICPs should be allocated in the thread.

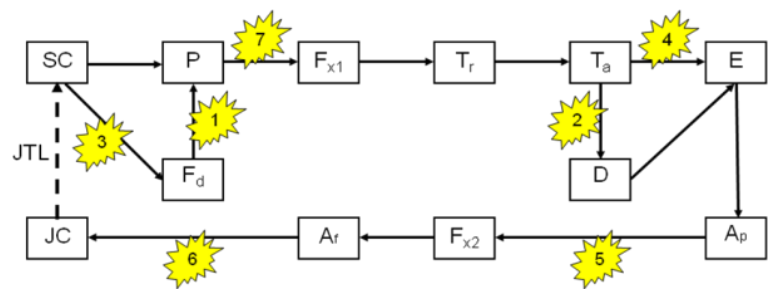

Fig. 3. Simplified TCS sequence for OMT \#14 (annotated)

In the Time Critical Strike (TCS) operational sequence, the Joint Force Command activity (JC) initializes the sequence by issuing the Joint Target List (JTL) to the Strike Command activity (SC). In a deliberate strike mission the SC activity might then execute a Prioritize Targets activity $(\mathrm{P})$ then task sensors to Find $\left(F_{d}\right)$ the assigned targets. However, in TCS the $\mathrm{F}_{\mathrm{d}}$ and $\mathrm{P}$ activities are executed concurrently, as indicated by the two paths to $\mathrm{P}$ in Fig. 3. The sensors must report detections and data not just on the target list but also from detections of unknown targets, which may become candidates for TCS. Once the target list has been updated and prioritized by the $\mathrm{SC}$, additional sensors must be tasked to identify and geolocate the targets. This comprises the Fix activity $\left(F_{x}\right)$. Targets then enter the Track activity $\left(\mathrm{T}_{\mathrm{r}}\right)$ through subsequent detections and predictions of target motion.

In the Targeting activity $\left(\mathrm{T}_{\mathrm{a}}\right)$, platforms and weapons are paired with the nominated targets. For a TCS target, platforms and weapons will be re-assigned from the original mission plan. The airspace for their ingress to targets must be Deconflicted (D) before the target is Engaged (E). After the engagement, the weapons platform makes a report and a preliminary Assessment $\left(\mathrm{A}_{\mathrm{p}}\right)$ of the outcome is made. SC may task a sensor capable of supporting identification to revisit the target, which is a second execution of the Fix activity. When the Fix activity is executed twice for different purposes, it is given different variable names, as indicated by $F_{x 1}$ and $F_{x 2}$ in Fig. 3. The operational sequence ends when a final Assessment $\left(\mathrm{A}_{\mathrm{f}}\right)$ of the outcome is made and reported to the JC activity, which uses the report to update the next issue of the JTL.
2) Allocation of ICP Bundles to OMTs: The next step in the analysis was to decide which ICPs to allocate to each gap. A detailed interoperability analysis of the systems was performed for each gap. The determination of information needs was just one aspect of the analysis. For some gaps, multiple ICPs would need to be implemented; for others one would be sufficient. The collection of ICPs that filled all the identified interoperability gaps for a specified OMT end-to-end was referred to as an ICP bundle.

In the case of OMT \#14, there were 7 interoperability gaps to be filled to enable TCS mission capability. The ICPs allocated to the 7 gaps for OMT \#14 are listed in Table I, which also shows the associations of the ICPs with the gaps (yellow firecrackers in Fig. 3). A close examination reveals that several of the ICPs are repeated. This is because some could be used for more than one gap. When the duplications are removed, the ICP the bundle is uniquely defined. The bundle allocated to the 7 interoperability gaps for OMT \#14 contained 22 ICPs. This was Bundle \#13.

TABLE I ALLOCATION OF ICPS TO INTEROPERABILITY GAPS

\begin{tabular}{|c|c|c|}
\hline Firecracker & ICP \# & ICP Title \\
\hline \begin{tabular}{|l|l|}
1 \\
\end{tabular} & TM97-077 & J15.0 Changes \\
\hline 1 & TM 92-035 & Points Revision \\
\hline 2 & TJ -491 & Add ABN LAND SURV to PLTFM/AST \\
\hline 2 & TJ93-001 & Air Control Revision \\
\hline 2 & TA99-001 & Align Drop Track Filter Protocols \\
\hline 2 & T.J93-054 & ASO (Strike) Revision \\
\hline 2 & TM97-021 & IFF/SIF Valid ity \& Currency Criteria \\
\hline 2 & T.J98-142 & J 12.6E0 Min Imp for SID =5 \\
\hline 2 & TM97-077 & J15.0 Changes \\
\hline 2 & TM94-053 & Mode IV Conflict Deletion \\
\hline 2 & TJ95-004 & NonC2 to NonC2NPGNetNumber \\
\hline 2 & TM 92-035 & Points Revision \\
\hline 2 & TM97-113 & Prohibit U nk/Pndg in $\mathrm{CDO}$ \\
\hline 2 & TJ90-003 & R2 for Inactive IU's Tracks \\
\hline 2 & TJ J90-101 & R2 Protocols \\
\hline 2 & TJ J94-030 & Spec Type in Surveillance Msgs \\
\hline 2 & TM97-020 & TextAddition to $J 7.3$ \\
\hline 2 & TJ97-143 & TPQ to TQ Mapping \\
\hline 2 & TJ $94-060$ & U se of PPLI TN \& ID Indic ator \\
\hline 3 & TJ J93-054 & ASO (Strike) Revision \\
\hline 3 & TJ98-063 & Delete Single Tgt App for J12.0 MADs $37 \& 38$ \\
\hline 4 & TJ93-054 & ASO (Strike) Revision \\
\hline 5 & TJ J93-054 & ASO (Strike) Revision \\
\hline 5 & TJ98-063 & Delete Single Tgt App for J12.0 MADs $37 \& 38$ \\
\hline 5 & TJ92-078 & Drone/RPV Translations \\
\hline 5 & TM97-004 & Land Platfom/Activity Add'ns \\
\hline 5 & TJ92-091 & M.14 Translation \\
\hline 5 & TJ J90-065 & WES Translations \\
\hline 6 & TJJ92-091 & M.14 Translation \\
\hline 6 & TJ90-065 & WES Translations \\
\hline 7 & TJJ93-054 & ASO (Strike) Revision \\
\hline 7 & T.J98-063 & Delete Single Tgt App for J12.0 MADs $37 \& 38$ \\
\hline
\end{tabular}

The interoperability gaps in each of the 39 OMTs were examined in this way. Each time a bundle was identified, the assessment team applied it to the other 38 OMTs to determine how many other OMTs the bundle enabled. The specification of subsequent bundles could include some or all of the previous collection of ICPs. Due to time limitations the process was limited to the specification of 15 bundles. The end result was a table with 39 rows (one for each mission thread) and 15 columns (one for each ICP bundle allocated as an end-to-end solution for a single OMT), which is depicted in Table II and displays assessment of the bundles across the OMTs. Bundle \#13 and OMT \#14 are called out by the loops in the table. 
The table was formatted to complement the color-coded NCTSI spreadsheet. A cell in the table was color coded 'green' if the given bundle (column in the table) filled every identified gap in the designated OMT (row in the table). Otherwise the cell was 'white'. The OMTs were used as the basis for a capability metric. If there were no preference between OMTs, the metric could as simple as the percentage of green cells in the column corresponding to an ICP bundle.

The user community preferred to rank the OMTs. For this case, the capability metric was taken to be the percentage of ranked OMTs whose gaps were filled by a specified ICP bundle. This metric was referred to simply as ' $\%$ Thread Coverage'. A score of $2.6 \%$ (i.e. 1/39) would indicate that the ICP enabled only one OMT, but it was the most important one.

TABLE II

ASSESSMENT OF ICP BUNDLES AGAINST MISSION THREADS

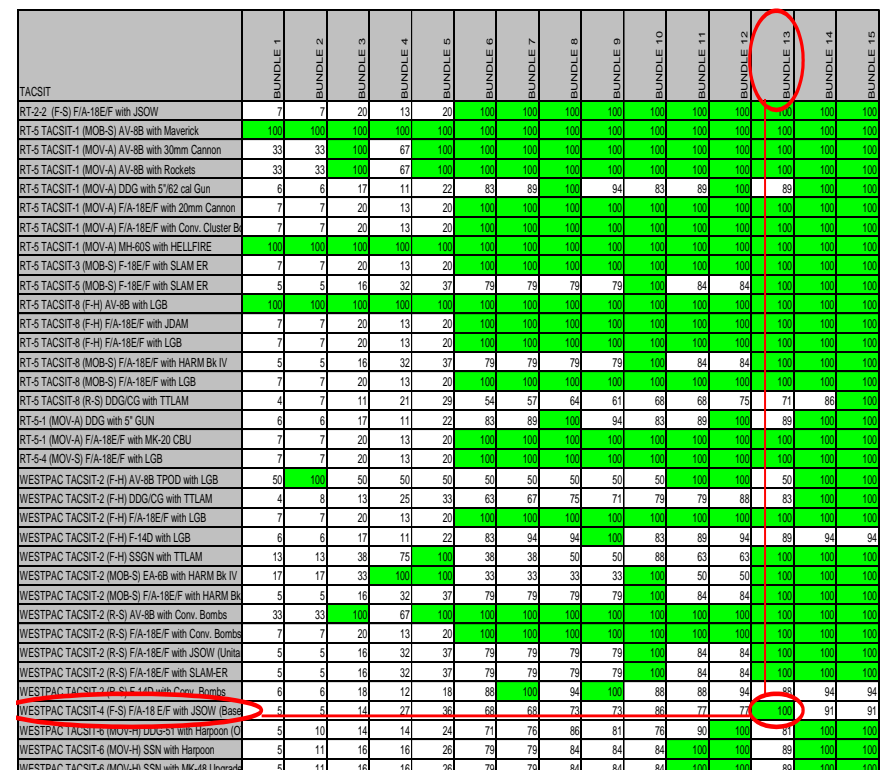

3) Capability tradeoff against cost at the SoS level: Each bundle was also associated with its cost. Therefore, an ordered pair of metric values (cost, \% thread coverage) was assigned to each bundle and the results were plotted to yield a conventional graph for cost-benefit tradeoff, as illustrated in Fig. 4. The distinction of this graph is that the benefit metric is not just for a single system but for a mission capability.

In the capability based tradeoff analysis, the $50 \%$ capability rank corresponded to bundle\#7, which had an estimated cost of $\$ 413 \mathrm{~K}$ to implement, as indicated in Fig. 4. (Note: Bundle Number is not a cost rank. Bundle \#7 was the $6^{\text {th }}$ bundle as ranked by cost). The cost was within the constraint budget. This recommendation was integrated with the results of the NCTSI Link 16 trade study to support the final procurement decision. The selection of this bundle also met the study objective of the requirements based approach, to specify a collection of ICPs that filled a broad range of interoperability gaps.

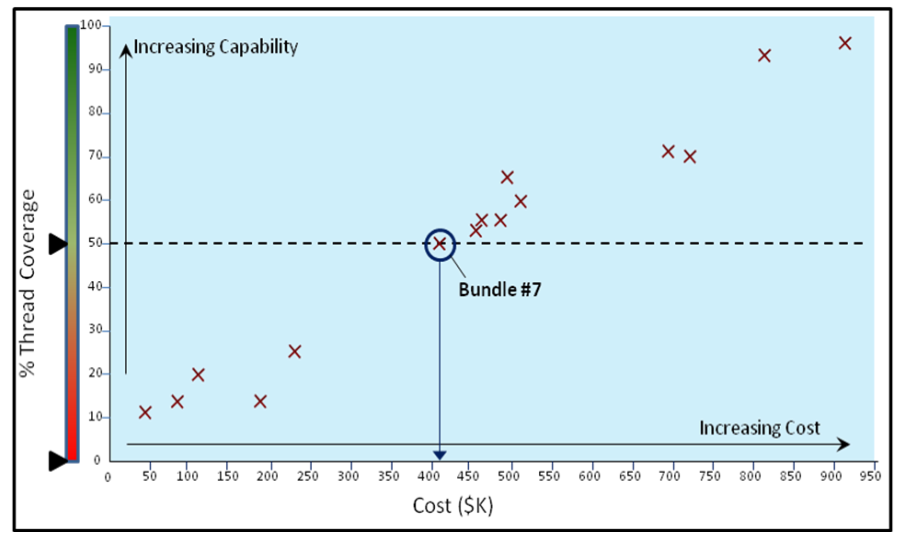

Fig. 4. Capability Cost-benefit Tradeoff for ICP Bundles

\section{Assessment of the legacy approaches}

Each of the two approaches made a unique contribution to the overall trade study but each also had deficiencies. Furthermore, neither approach was repeatable.

1) Requirements Based Approach: This approach illustrated the challenge of trying to use traditional single system requirements based tradeoffs analyses in a CBA environment. Although the approach did align the ICPs to operational requirements, it lacked methods for aligning requirements to the operational capabilities. It could not be guaranteed if a certain combination of requirements were met that a specified operational capability would be enabled. Furthermore, there were no methods for organizing the ICPs into portfolios. The spreadsheet analysis provided a useful starting point, but final decisions ultimately rested upon engineering and operational judgment, and pattern recognition in the spreadsheet. Consequently this was not a repeatable approach.

2) Capabilities Based Approach: This approach successfully used OMTs to model instances of operational capability. This was a significant innovation. The allocation of bundles of ICPs to these end-to-end mission threads showed how to organize the ICPs to enable mission capabilities. The concept of using the mission thread coverage as a metric to aggregate SoS performance as operational capability, provided a strong connection with a traditional tradeoff analysis that could be visualized in a cost-benefit graph at the capability level, such as Fig. 4. However, there was at best a loose connection between this representation of mission capabilities and the specifications of operational requirements.

Despite the broad interest this approach generated at the time of the NCTSI study, it was never successfully repeated by the rest of the community of interest. It is not known where the approach broke down when other parties considered using it. Although a prescriptive description of the approach was never written down, the sequence of architecture artifacts and analysis products summarized here in Section II part B seems to be a logical and intuitive approach. The problem with the 
repeatability of the approach may have been that the engineering analysis became entangled with the selection decision of allocating ICPs. This is borne out by how labor intensive the analysis effort was. In response to a query from DASN IWS, it was estimated that the labor cost of the analysis was equivalent to a substantial fraction of the cost of the acquisition of the ICP bundle that was procured.

\section{RELATIONAL ORIENTATED APPROACH}

Relational orientation will be applied to complete and make precise the approach attempted in the original capability based tradeoff analysis. The metamodel created from the relation oriented approach is an abstraction from which models can be instantiated. This will provide a framework structure to navigate through the artifacts of the SoS level analysis and tradeoffs in a repeatable way. In the original study the interoperability analysis was performed for each ICP considered for allocation to an OMT. This approach entangles the solution for information needs with the interoperability analysis. The selection of ICP bundles can be done more efficiently by concentrating on the information needs before performing the interoperability analysis. Using the metamodel focused on the $\mathrm{J}$-series messages results in a trade-off and interoperability analysis performed on a smaller set of ICP bundles. Relational orientation will use the metamodel to instantiate a series of models and relational transformations, which can be used to specify candidate ICP bundles.

There is sufficient information in Section II to populate the framework structure of the metamodel in the simplified case study of the original tactical data link interoperability gap presented in Section B. The navigation of the frames and transformations specified in this section then provides a proof of concept demonstration for the ROSE approach to the SoS interoperability gap. The complete engineering details associated with the frames are available through official sources but the level of detail needed to actually replicate the original tradeoff analysis is beyond the scope of a technical paper. (Recall that the SV-6 data base had 2,857 lines of pointto-point interoperability information in a document based environment.)

\section{A. Relational Transformation}

This section briefly reviews the details of the binary and the unary relational transformations that will be used and where they fit into the metamodel and analysis approach; reference [12] provides further detail.

Binary transformations will be used to formalize the OMTs used in the MCPs and for architecture analysis. OMTs as used in the legacy CBA approach are generally regarded as operational sequences to which mission resources have been allocated [13]. Because an OMT can be represented as a source model with a target model, and an association between the two, this can be the basis for specifying a framework similar to Fig. 1 comprised of: (i) a model of the operational sequence (based on the precedence order of the operational activities), (ii) the set of systems from the SoS allocated to the operational sequence, and (iii) an allocation matrix (transformational association) that induces a transformation of the operational sequence model into the SoS. The ordering relationships transformed from the operational sequences onto the systems allocated from the SoS expose the collaborations and sequencing between the systems.

Given a model $\mathbf{M}$ with elements in the set $M$, a mathematical binary relation $\mathrm{R}$ on $M$ is a defined collection of ordered pairs of elements taken from $M$, i.e. $\left(\mathrm{y}_{\mathrm{i}}, \mathrm{y}_{\mathrm{j}}\right) \in \mathrm{R}$ where $\mathrm{y}_{\mathrm{i}}, \mathrm{y}_{\mathrm{j}} \in M$. The equivalent notation $\mathrm{y}_{\mathrm{i}} \mathrm{Ry}_{\mathrm{j}}$ is also used.

Let $\mathbf{N}$ be another model with elements in $N$ and with binary relation $\mathrm{S}$ on $N$. A binary transformational association $\mathrm{Q}$ between $\mathbf{M}$ and $\mathbf{N}$ is a collection of ordered pairs of elements taken from $M$ and $N$, i.e. $\left(\mathrm{y}_{\mathrm{i}}, \mathrm{x}_{\mathrm{k}}\right) \in \mathrm{Q}$. The element $\mathrm{y}_{\mathrm{i}} \in M$ is said to be associated with the element $\mathrm{x}_{\mathrm{k}} \in N$ by $\mathrm{Q}$. The equivalent notation $\mathrm{y}_{\mathrm{i}} \mathrm{Qx}_{\mathrm{k}}$ is also used.

The calculation of the transformation of binary associations is straight forward:

$$
\left(\mathrm{y}_{\mathrm{i}}, \mathrm{y}_{\mathrm{j}}\right) \in \mathrm{R} \text { with }\left(\mathrm{y}_{\mathrm{i}}, \mathrm{x}_{\mathrm{k}}\right),\left(\mathrm{y}_{\mathrm{j}}, \mathrm{x}_{\mathrm{l}}\right) \in \mathrm{Q} \text { implies }\left(\mathrm{x}_{\mathrm{k}}, \mathrm{x}_{\mathrm{l}}\right) \in \mathrm{RQ}
$$

If RQ is a subset of one of the binary relations on $\mathrm{N}$, e.g. $\mathrm{RQ}$ is a subset of $\mathrm{S}$, then $\mathrm{Q}$ is said to induce a weak relational transformation $\mathbf{M} \rightarrow \mathbf{N}$. This is the type of transformation that was illustrated in Fig. 1. Frames $\underline{\mathrm{M}}$ and $\underline{\mathrm{N}}$ for the graphical models depicted in the figure are represented as the incidence matrices of the graphs and the transformational frame $Q$ is represented by the matrix of associations between the nodes. The formula for calculating binary transformation asserts that the association of the nodes between the graphs also implies a transformation of the edges. This formula should not be confused with ordinary matrix operations such as matrix multiplication. In algebraic graph theory, if the associations made by $Q$ were single-valued then $Q$ would be a graphical homomorphism.

Unary transformations will be used for allocating the ICPs in a repeatable way to the OMTs and for analysis. This type of transformation associates subsets (domains) between models. If $\mathrm{Q}$ associates $M$ with $N$ then given a subset $R$ of $M$, define $R \mathrm{Q}=\{\mathrm{x} \in N: \mathrm{yQx}$ for some $\mathrm{y} \in R\}$. This is the 'natural' subset of $N$ that $\mathrm{Q}$ associates with the subset $R$ of $M$. It will be referred to as the unary transformation of $R$ by $\mathrm{Q}$.

When elements of two models $\mathbf{M}$ and $\mathbf{N}$ are associated in a table, the rows correspond to elements of $M$ and the columns correspond to $N$. The associations in the body of the table then become a unary transformation $\mathrm{Q}$, which associates subsets of the model elements that define the rows of the table with corresponding subsets of the elements that define the columns. 


\section{B. Metamodel for the Proposed Approach}

The metamodel depicted in Fig. 5 is comprised of three structures: Information, Interface, and Capability; frames for operational requirements and mission objectives are also included. The elements of the metamodel are the relational frames of the structures. Transformations between the frames are the lines between the elements in the metamodel. Altogether eleven frames must be specified:
F1: J-series message (J-msg)
F2: Information element (IE)
F3: System function (SysFctn)
F4: Interface change proposal (ICP)
F5: Node
F6: System (of systems)
F7: Operational capability (OpCap)
F8: Operational sequence (OpSeq)
F9: Operational task (OpTask)
F10: Operational requirement (OpReq)
F11: Mission objective (MsnObj)

The first three frames and the transformations between them form two frameworks that comprise the Information structure. The next three frames form the Interface structure and the three operational frames together with the shared Node frame are used for the Capability structure. The remaining two frames in the metamodel are related to requirements.

In this capability based ROSE approach, an information centric viewpoint will be taken. The flow of information needs through the metamodel (indicated by the dashed line wrapping around the outside of the metamodel) together with unary and binary relational transformations can be used to generate candidate bundles of ICPs for the SoS level tradeoff analysis.

The three dash-dot lines in the metamodel indicate the three allocation associations that are the trade space for the study. The solid lines are fixed relationships. The requirements based approach in the legacy analysis of Section II allocated ICPs to the nodes in order to satisfy the specified operational requirements. The capabilities based approach allocated nodes to specified operational capabilities and then used the associated allocations of systems to operational tasks (using system functions) to satisfy capability needs.

Operational capability will be modeled by an OMT which associates an operational sequence $(\mathrm{OpSeq})$ with one or more nodes. The allocation of systems to the sequence, which is done using system functions (SysFctn) and operational tasks (OpTask), then populates the OMT which subsequently can be assessed as to whether it satisfies the mission objectives. This captures the ways and means portion of the DoD concept of operational capability [14]. When the OMT is informed by a scenario, conditions and standards, it then becomes a model of an instance of operational capability.
The system functions ideally should be specified in a standardized way so that the input details and the output details of the function are explicitly known and standardized. Thus, the system functions would be implementation free and the J-series messages needed would be independent of the system implementing the function. If a list of standardized functions is not available the systems can be used directly, but the solution will not be implementation free.

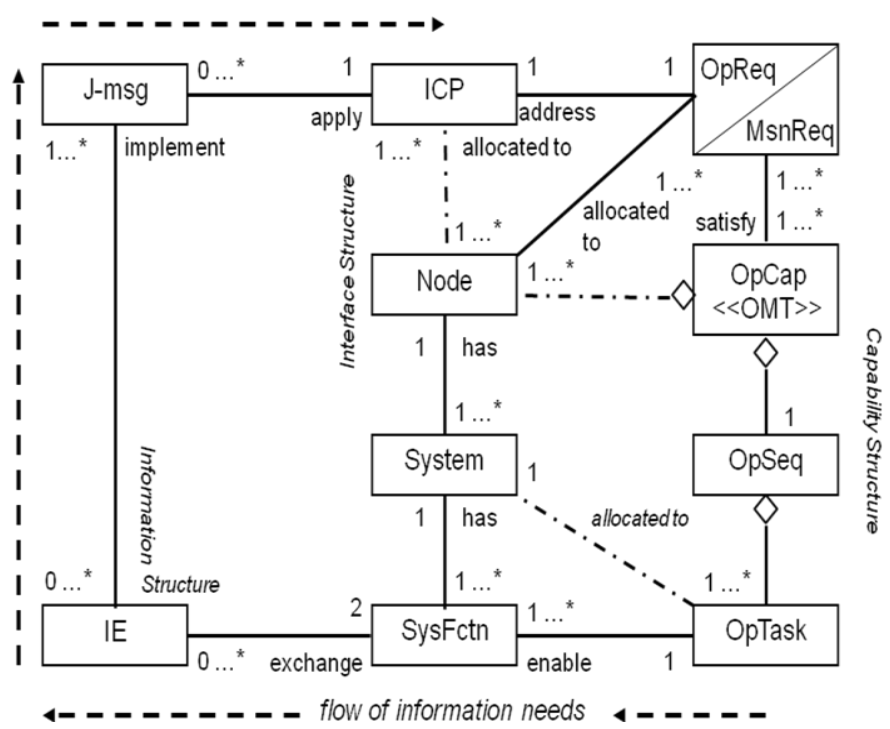

Fig. 5. Metamodel for the ROSE Approach

In the previous Link 16 analysis the allocation of ICPs to OMT problems was based on interoperability analysis. The analysis became entangled with the selection decision of allocating ICPs. This type of ad hoc approach contributed significantly to the labor intensive nature of the legacy capabilities based approach.

For the metamodel in Fig. 5 an 'interoperability gap' will mean that the information needs of an operational task are not being satisfied. Candidate ICPs will be identified to fill the gap by tracing from the operational task with the need through the Information structure to identify the J-series messages that will fill the gap and the ICPs that apply the messages. Detailed interoperability analysis can then be performed as required on the ICPs allocated to a bundle after navigating the Interface structure. This disentangles the detailed interoperability analysis from ICP selection decisions. The factorization of the metamodel into three structures also organizes knowledge of the SoS into architectural domains individually aligned for verification and validation of the proposed solutions.

The metamodel for the ROSE approach and its factorization into Information, Interface, and Capability structures, permits the easily calculated generation of ICP bundles using unary transformations, $E^{\tau} \rightarrow J^{\tau} \rightarrow I^{\tau}$, by navigating through the Information structure as indicated in Fig.5. The frames $E^{\tau}, J^{\tau}$, and $I^{\tau}$ are the frames representing the domains of IEs, J-series messages, and ICPs, respectively for a specified OMT indexed by $\tau$. 


\section{Populating the Frames of the Metamodel}

This will section will instantiate the requisite frames and relational transformations specified the metamodel. The detailed information provided in Section II can only be used to partially populate the frames; but this will be sufficient to precisely replicate results from the legacy capability based approach on a reduced demonstration set of the ICPs. Specifically, a subset of ICPs analyzed in the legacy Link 16 interoperability gaps identified for OMT \#14 will be used to trace the information needs determined by the Capability structure back through the Information structure to the J-series messages using unary transformations; and then to the ICPs in order to specify an ICP bundle. Thus, $E^{14} \rightarrow J^{14} \rightarrow I^{14}$ will yield a partial solution through population of the frames.

\section{1) Sources for the demonstration data set}

The CBA analysis identified 76 ICPs that could be mapped to J-series messages. The open source summary of the study [3] documented a limited set of the data used in the study. The summary was organized around what was originally called Mission Thread \#14 which was populated by 27 operational activities organized around the $\mathrm{F}^{2} \mathrm{~T}^{2} \mathrm{EA}$ sequence: 6 platform nodes, 6 communication networks, 18 systems with their associated system functions (specified numerically), and 7 identified interoperability gaps. The ICP Bundle (\#13) in the CBA analysis specified 22 ICPs that were assessed to be sufficient to fill the 7 interoperability gaps based on their application of $\mathrm{J}$-series messages.

Rows 24 - 44 in the NCTSI spreadsheet as depicted in Fig. 2 called out 11 ICPs associated with one or more of $18 \mathrm{~J}$-series messages. Of these 11 ICPs only 4 belonged to Bundle \#13 as specified by the CBA analysis in Table I. Although this data from the original mission thread and the spreadsheet are sparse, they are sufficient to precisely replicate the selection of these 4 ICPs. Details of the data are provided in a series of tables throughout this Section.

\section{2) Information needs from the Capability structure:}

The objective of this section is to specify a subset of IEs from $E^{14}$ needed for the gaps in OMT \#14 based on the partial population of frames using detailed information provided in Section II. Table III is an integration of multiple frames and associations that model the operational flow and the associations of the nodes and systems with the information needed by the Capability structure. (Note: Definitions of acronyms can be found at the end of this paper).

The matrix on the left side of the Table specifies the OpSeq frame that represents the graphical model of the operation sequence in Fig. 3. It has 13 ordered pairs of activities. A tick mark in the $(\mathrm{m}, \mathrm{n})$ cell means that the activity in row $m$ directly precedes the activity in column $n$ in the sequence, e.g. $\mathrm{T}_{\mathrm{a}}$ directly precedes both $\mathrm{D}$ and $\mathrm{E}$. The parenthetical tick mark for JC and SC is the initialization of the loop.
TABLE III

TRANSFORMATIONAL FRAMES: ALLOCATION OF NODES TO OPERATIONAL SEQUENCE AND SYSTEM FUNCTIONS TO TASKS

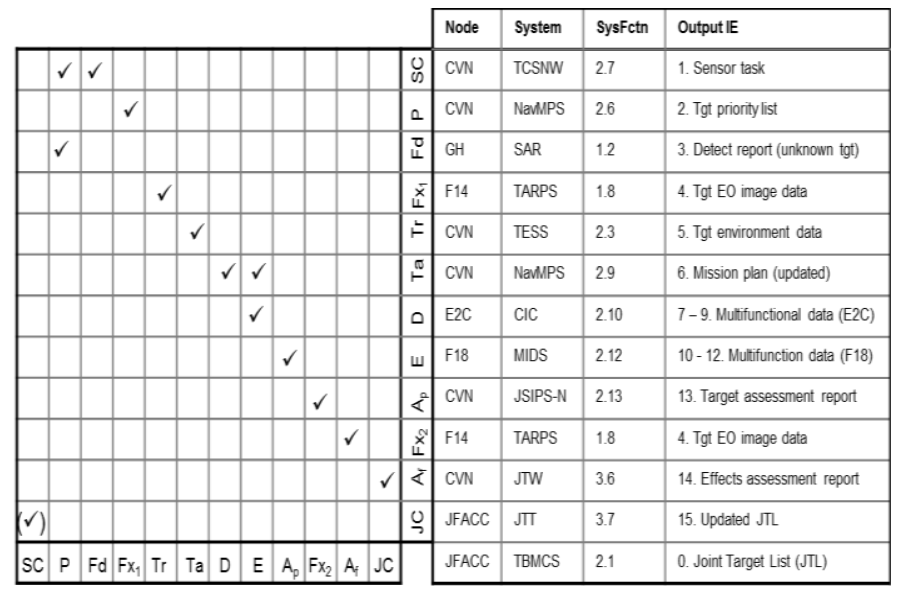

The allocations in the Capability structure (displayed in the right hand side of the metamodel in Fig. 5) are summarized in the right hand side of Table III. The operational sequence is implemented by allocating one of 6 physical nodes to each of the 12 operational activities. Some nodes, such as the CVN, are allocated to more than one activity. As with the multiple instantiations of the activities in Section IIB, multiple instantiations of a node must be distinguished if sequencing is to be preserved. The pairing of activities and nodes gives a binary relational transformation of the graph in Fig. 3 (modeled by the frame in Table III) into the graph in Fig. 6, which mirrors the order of instantiation of activities specified in Table III and the allocation of nodes to activities in the operational sequence. Therefore, Fig. 6 models the flow of the IEs through the allocated SoS.

The 6 nodes employ 11 systems, as indicated in Table III, and each system executes one of the system functions called out in the OMT depicted in Fig. 6. The system functions are named by a single decimal number and each produces one or more of the 15 output IEs called out in Table III. The system names and system functions have also been mapped onto the nodal sequence in Fig. 6. Interoperability exists at the nodal level [9]. Therefore the 7 interoperability gaps called out in the figure are associated with nodal pairs. This leads to a pairing of the SysFctns in which the target SysFctn needs IEs from the source SysFctn. Therefore each of the 7 interoperability gaps is associated with a SysFctn pair and an IE that is not being properly provided.

In each of these gaps, the requisite IEs can be found using Fig. 6 and Table III. First Fig. 6 is used to identify the source system that must provide the IE to the target system. Table III can then be used to identify the SysFctn pair involved and the IEs be provided from the source system. The following narrative illustrates this process for OMT \#14 in Fig. 6. The results are summarized in Table IV following the narrative. 


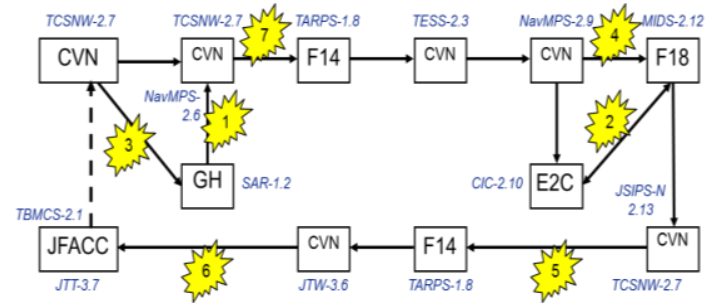

Fig. 6. OMT \#14 TCS Nodal Sequence (annotated)

The 7 gaps identified fall into three general groups: tasking sensors, reporting, and exchange of mission data. Of particular interest in a TCS mission is the detection of unknown targets.

There are three interoperability gaps in Fig. 6 for sensor tasking: 'Firecrackers'\#3, 5, and 7. First (\#3) is the CVN tasking of the Global Hawk $(\mathrm{GH})$ synthetic aperture radar (SAR), for TCS target detections. Next (\#7) is the CVN tasking of the Tactical Airborne Reconnaissance Pod System (TARPS), carried by the F-14 fighter aircraft, for imagery to support the Naval Mission Planning System (NavMPS) on the CVN for target identification. Last (\#5) is the CVN tasking of the F-14 TARPS for imagery to be used, by the Navy Joint Surveillance Imagery Processing System (JSIPS-N), for damage assessment.

There are two interoperability gaps in Fig. 6 for reporting: 'Firecrackers'\#1 and 6. First (\#1) is the GH sending detection reports from its SAR radar to the NavMPS. Last (\#6) is the SC activity aboard the aircraft carrier node (CVN), using the Joint Targeting Workstation (JTW), to make a weapons effects assessment report to the Joint Forces Air Component Commander (JFACC), who must update the JTL.

The remaining two gaps ('Firecrackers'\#2, 4) relate to the exchange of mission data for an F-18 strike aircraft responding to an updated order to engage a TCS target. In Fircracker\#4, the SC activity aboard the CVN must give the F-18 an updated mission plan from the NavMPS. The communication gap (\#2) between the $\mathrm{F}-18$ and the $\mathrm{E}-2 \mathrm{C}$, which is an airborne surveillance and $\mathrm{C}^{2}$ aircraft, is more complicated and will be split into two gaps. First (\#2a) the F-18 needs both navigation data (for vectors to the target and airspace deconfliction) and targeting data. Multifunction data (IEs 7 -9) can be allocated as follows: IE7 (Airspace de-confliction ) can be supported by air track; IE 8 (Targeting Data) can be supported by surface track of the target, target/track correlation, and target bearing; navigation data from the $\mathrm{E}-2 \mathrm{C}$ is provided in the form of flight vectors, which is IE 9. These data are sent to the Multifunction Information Distribution System (MIDS) on the F-18, which is a communication component in Link 16. Next (\#2b), multifunctional Data (IEs 10-12) can be allocated as follows: IE10 can be supported by the air precise participant location and identification (PPLI); IE11 is data to be sent from the F-18 to the E2C with regards to its status condition; and IE12 is supported by a mission update from the F-18.

Table IV determines the subset of $\mathrm{E}^{14}$ that will be used to trace through the Information structure: $\{$ IE1, IE3, IE6, IE7, IE8, IE9, IE10, IE11, IE12, IE14\}.
TABLE IV

TRANSFORMATIONAL FRAME: IE ALLOCATION TO SYSTEM FUNCTION PAIRS BASED ON INFORMATION NEED (OMT \#14)

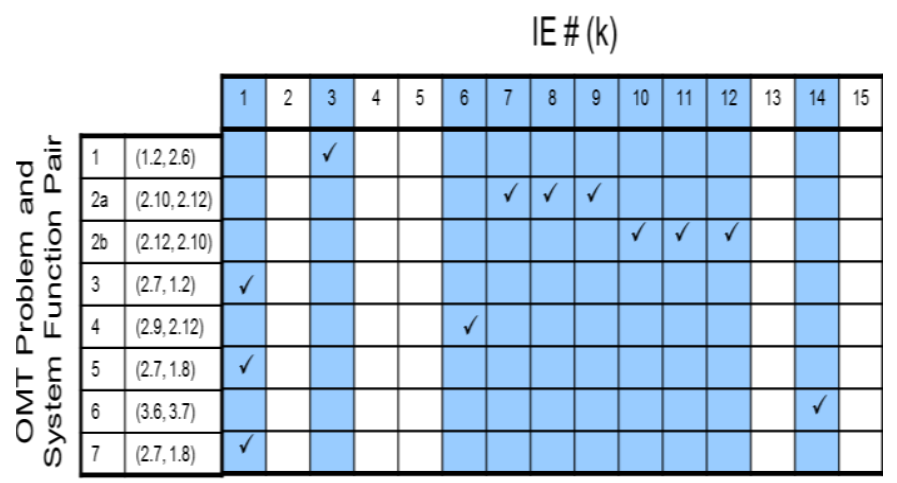

3) Transformation of $E^{14} \rightarrow J^{14}$ : The results of the transformation represented by Table IV will be transformed into $\mathrm{J}$-series messages by associating the messages with the IEs that they implement. These $18 \mathrm{~J}$-msgs from the NCTSI spreadsheet in Fig. 2 will be used as the subset $J^{14}$. Table V lists and indexes these messages, gives their J-numbers, and associates them with the IEs based on information content. The resulting transformational frame is represented by Table VI. When the transformation is applied to subset of $E^{14}$ determined by Table IV, the result is the subset of J-msgs that will be used to create the ICP bundle for OMT \#14. These messages can be seen by the shading in Table VI.

4) Transformation of $\mathrm{J}^{14} \rightarrow \mathrm{I}^{14}$ : The final step for allocating ICPs to OMTs is the calculation of the unary transformation of the subset of messages $J^{14}$ into the set $I$ of all ICPs to obtain $I^{14}$. The set $J^{14}$ is determined by the transformation represented by Table VI. The tick marks in Table VI are the 22 associations of J-msgs with IEs called out in Table V. The 10 IEs needed for OMT\#14 are identified as the subset of shaded columns in Table IV, which are transferred to Table VI to set a unary transformation of IEs into J-msgs: every J-msg with a tick mark in a shaded column is then selected for OMT\#14. The subset $J^{14}$ is then determined to be: $\{\mathrm{J} 2, \mathrm{~J} 3, \mathrm{~J} 4$, J5, J6, J11, J12, J13, J14, J16, J17\}.

TABLE V

ASSOCIATION OF IEs WITH J-MSGs FOR OMT\#14

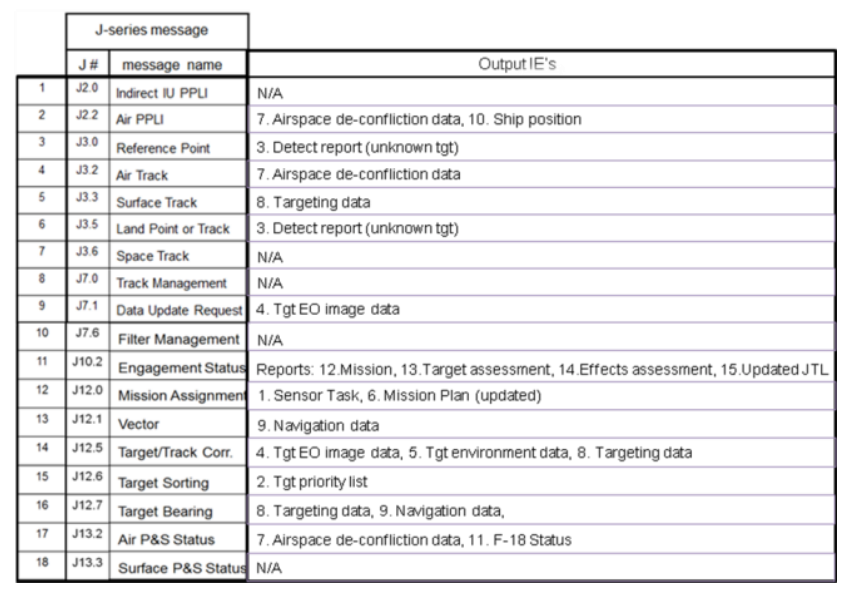


TABLE VI

UNARY TRANSFORMATION OF IEs TO J-MSGS (OMT \#14)

$$
\text { IE \# (k) }
$$

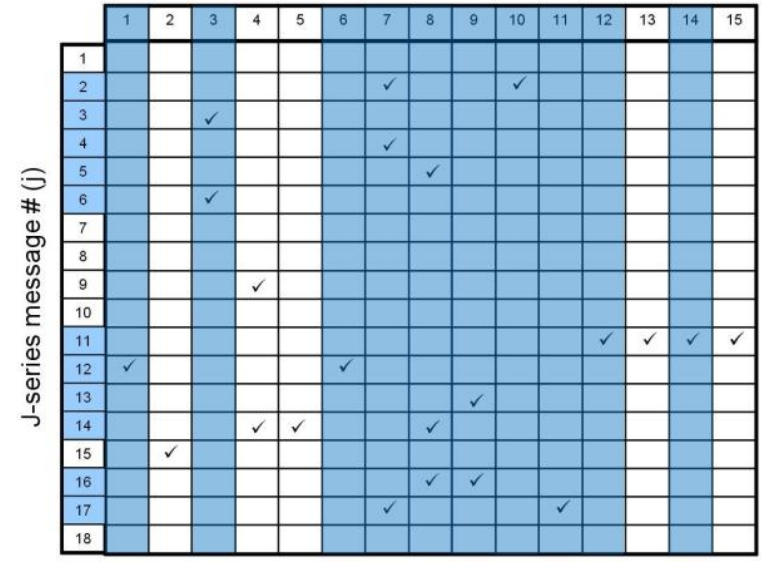

Table VII displays the 11 ICPs from the NCTSI spreadsheet shown in Fig.2. This will be used as the partial population of $I^{14}$ for identifying the four ICPs belonging to Bundle \#13.

TABLE VII

ICP FRAME: PARTIAL LIST OF ICPS

\begin{tabular}{|c|c|c|c|}
\hline & \multicolumn{3}{|c|}{ ICP (TJ \# or TM \#) } \\
\hline 1 & TJ91-109 & & \\
\hline 2,3 & TJ92-002 & TJ92-091 & \\
\hline $4-6$ & TJ93-001 & TJ93-054 & TJ93-096 \\
\hline 7 & TJ96-001 & & \\
\hline 8,9 & TM92-035 & TM92-047 & \\
\hline 10,11 & TM93-003 & ТМ93-067 & \\
\hline
\end{tabular}

Table VIII displays the association of J-msgs with ICPs from the NCTSI spreadsheet in Fig. 2 for the subset $J^{14}$. Applying the unary transformation in this frame results in the ICP subset indicated by the shaded columns. This is the ICP bundle that the ROSE methodology calculates, for satisfying the information needs for the interoperability gaps in OMT \#14, based on the partially populated frames of the demonstration data. The transformational frames represented by Tables IV and V can also be used to identify the J-msgs allocated to the 7 individual gaps in OMT \#14.

TABLE VIII

UNARY TRANSFORMATION OF J-MSG BUNDLE INTO ICP BUNDLE (OMT \#14) ICP \# (i)

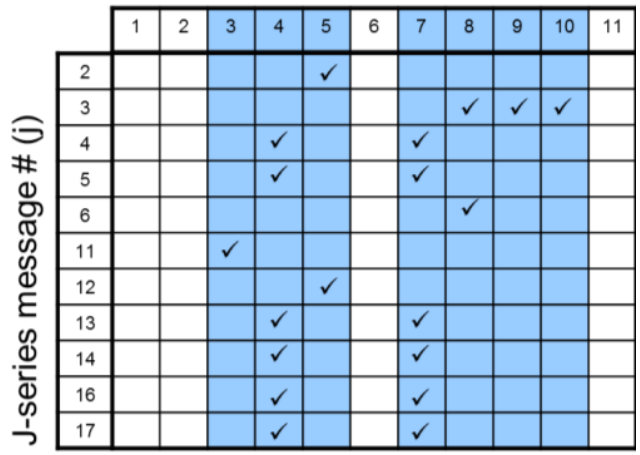

A close inspection of this bundle of 7 ICPs reveals that there are redundancies, i.e. the message set $J^{14}$ can be applied by fewer than all 7 of the ICPs. This can be seen from the multiplicity in Table VIII: when there is more than one tick mark in a row, the message in that row can be applied by more than one ICP. The multiplicity is greater than 1 except for $\mathrm{j}=$ 2, 6, 11, 12. The ICPs associated with these four J-series messages in order of transformation are: $\mathrm{i}=5 ; 8 ; 3 ; 5$. This reduces to $\mathrm{i}=3,5,8$. Because the multiplicity is 1 , these are 'must have' ICPs. These 3 ICPs actually apply 5 of the 11 messages $(j=2,3,6,11,12)$. The other 6 messages $(j=4,5$, $13,14,16,17)$ can all be applied by a single ICP, e.g. $i=4$.

5) Comparison of ICP bundles for OMT \#14: The demonstration that ROSE correctly replicated the same results as the legacy CBA analysis is now straight forward. The reduced bundle for OMT\#14 with no redundancies can be formed using $\mathrm{i}=3,4,5,8$. Referring to Table VII, these are TJ92-091, TJ93-001, TJ93-054, and TM92-035. These 4 ICPs are precisely the ICPs that the partial list of ICPs in Table VII has in common with the 22 ICPs in Bundle \#13 in the legacy tradeoff analysis. (See Table I.) Therefore, the ROSE approach has precisely replicated the partially populated capability based solution for OMT \#14.

\section{Verification and Validation of ICP Bundles}

The ROSE implementation of a capabilities based approach inherently provides a significant level of verification through its use of the Capability framework structure, to include OMTs. Thus, by working with the operational community, there is general agreement that the correct problem is being solved in advance of any engineering analysis. Similarly, the Interface framework structure captures much of the needed information for verification of the interoperability of the proposed solutions (ICP bundles). In particular, the use of system function pairs (or system pairs) exchanging information as the starting point for generating solutions identifies key points in the SoS architecture for initiating the verification analysis.

\section{E. Capability Tradeoffs at the SoS Level}

As before, the final step of the tradeoff analysis must ask what percent of the ranked OMTs does each bundle fix and how much does the bundle cost? The association of this percentage with cost for each bundle yields the same classic cost tradeoff graph as in the legacy capability based study.

\section{F. Relational Oriented Approach Summary}

The ROSE approach to the Link 16 tradeoff analysis is fully prescribed. It offers a metamodel that implements a capability based approach and has elements for integration with a requirements based approach. The execution of the approach prescribed in Section III, using the same data as in the legacy 
capability based approach, was able to precisely replicate results from the legacy study. This gives a proof of concept demonstration that the approach is repeatable.

The metamodel for the ROSE approach and it factorization into Information, Interface, and Capability framework structures permits the rapid and easily calculated specification of ICP bundles using unary transformations: $E^{\tau} \rightarrow J^{\tau} \rightarrow I^{\tau}$, to navigate through the Information structure. With careful attention to notation, it should be possible to apply the metamodels and transformations specified in the case study to a larger scale problem. Furthermore, the factorization of the metamodel into three structures disentangles the details of engineering analysis from the selection decision of candidate ICP bundle specification and organizes knowledge of the SoS into domains individually aligned for verification and validation of the proposed solutions.

\section{CHALLENGES OF CAPABILITY BASED ACQUISITION}

CBA is an approach to acquiring military systems of systems that has continued to evolve since early advances described in Section II. The central concept is the shift from acquiring individual systems based on functional requirements specific to each system, to mission level capabilities for the SoS of interest. Tradeoff analyses at this level have a complexity of much greater scope than just deciding between the alternative systems and performing interoperability assessments. This was accompanied by a shift in system engineering practice to a more architecture based approach [9]. In the US, legacy acquisition practices such as the US DoD 5000 were augmented by the Joint Capabilities Integration and Development System (JCIDS). Concurrently the UK introduced Through Life Capability Management (TLCM). Chapter 18 of [3] offers an introduction to and overview of JCIDS and TLCM.

Implementing a dramatic shift in acquisition approach such as CBA is accompanied by numerous challenges. The first is the complexity and ambiguity of the CBA problem makes it a wicked problem (see Chapter 21 of [3]) that resists the prescriptive and reductionist approaches of scientific methodology. For example, the core concept of capability is itself open to so many interpretations that agreement on the model and technical meaning of the concept is a challenge. CBA is also a portfolio problem for resource allocation, which could benefit from an extensive literature on portfolio decision analysis that goes beyond the acquisition of military systems. See, for example [16]. There are also more sophisticated figures of merit than the simple one used in the legacy CBA approach. The complexity of the CBA problem leads to increased costs for management and analysis as is evident the in the introduction of JCIDS in the US, TLCM in the UK, and the legacy CBA AoA tradeoff analysis presented in Section II. The entanglement of different decisions factors for capabilities, interfaces, and information needs in the legacy (and even the evolving) CBA approaches further exacerbate the costliness of the problem. Management of the requisite data for repeatable CBA portfolio analyses needs to be accomplished through appropriate databases, and repeatable methods for accessing the data, to create architectural artifacts for analysis and decision making, thus managing at the artifact level runs the risk of obsolescence of the artifacts for re-use.

\section{CONCLUSIONS}

The assessment of alternatives (AoA) for interoperability improvements to TDLs in a deployed SoS exhibits challenges associated with the general problem of capability based acquisition (CBA). The 2003 Link 16 tradeoff study offered two analysis approaches to the AoA problem for implementation of interface change proposals (ICPs); one was requirements based and the other capability based. Although delivering useful results, neither approach prescribed a method that was seen to be repeatable.

ROSE offers a structured approach that was used to develop a metamodel to implement a capability based approach and offer integration points for a requirements based approach. The metamodel addresses the wicked problem of CBA by factoring the mission level architecture into more manageable domains and thus providing a structure to navigate through the requisite architectural artifacts for the selection of bundles of ICPs. Capabilities are modeled by operational mission threads (OMTs) that can be assessed in specified scenarios. The approach prescribed by ROSE for 'portfolio selection' of the bundles was able to precisely replicate results from the legacy capability based approach on a reduced demonstration set of the ICPs.

The frames and transformations provided in the case study were specified and calculated for the specific data of the reduced demonstration set at a level of detail, to enable the reader to repeat the same results. Because the approach is not prescriptive to figures of merit, it should be possible to accommodate other portfolio decision analysis metrics. Frames and transformations prescribed by ROSE should be reusable to generate or modify architectural artifacts as necessary for further AoA studies; and if implemented in a modern modeling language should lend themselves to machine automation. Attention can then be focused on the management of the databases accessed to create the artifacts rather than management of the artifacts. Therefore the results presented in this paper not only resolves a long standing problem in TDL SoS interoperability, but also offer innovations that can be applied to the challenges of CBA.

The abstract approach employed should be applicable to a much wider class of problems than data link interoperability. While the ROSE approach to the Link 16 study exploited information needs to navigate the metamodel of Fig. 5, in more general problems once a domain model is defined, key specific 
elements exchanged between entities in the SoS can be used to efficiently guide portfolio selection in a rigorous and repeatable way. Thus the approach and results of this paper offer an early demonstration of a significant new methodology for SoS portfolio selection and evaluation.

\section{ACRONYMS}

\begin{tabular}{|c|c|}
\hline Abbrev & Meaning \\
\hline $\mathrm{A}_{\mathrm{f}}$ & Final Assessment \\
\hline$A_{p}$ & Preliminary Assessment \\
\hline AoA & Assessment of Alternatives \\
\hline C3 & $\begin{array}{l}\text { Communication and } \\
\text { command and control }\end{array}$ \\
\hline CBA & $\begin{array}{l}\text { Capability Based } \\
\text { Acquisition }\end{array}$ \\
\hline CIC & Combat Information Centre \\
\hline CVN & $\begin{array}{l}\text { Nuclear Aircraft Carrier } \\
\text { Node }\end{array}$ \\
\hline D & Deconflicted \\
\hline DASN & $\begin{array}{l}\text { Deputy Assistant Secretary } \\
\text { of the Navy }\end{array}$ \\
\hline DoD & Department of Defense \\
\hline E & Engage \\
\hline E2C & $\begin{array}{l}\text { Hawkeye Warning and } \\
\text { Control Aircraft }\end{array}$ \\
\hline $\mathrm{F}^{2} \mathrm{~T}^{2} \mathrm{EA}$ & $\begin{array}{l}\text { Find, Fix, Track, Target, } \\
\text { Engage, and Assess }\end{array}$ \\
\hline$F_{d}$ & Find \\
\hline $\mathrm{F}_{\mathrm{x}}$ & Fix \\
\hline GH & Global Hawk \\
\hline ICP & Interface Change Proposals \\
\hline $\mathrm{IE}$ & Information Elements \\
\hline IWS & Integrated Weapon Systems \\
\hline JCIDS & $\begin{array}{l}\text { Joint Capabilities Integration and } \\
\text { Development System }\end{array}$ \\
\hline JFACC & $\begin{array}{l}\text { Joint Forces Air Component } \\
\text { commander }\end{array}$ \\
\hline JSIPS-N & $\begin{array}{l}\text { Joint Surveillance Imagery } \\
\text { Processing System - Navy }\end{array}$ \\
\hline JTT & Joint Tactical Terminal \\
\hline JTW & Joint Targeting Workstation \\
\hline MCP & Mission Capability Package \\
\hline MIDS & $\begin{array}{l}\text { Multifunction Information } \\
\text { Distributed System }\end{array}$ \\
\hline
\end{tabular}

[2] R.C. Stephens, "Calendar Year 2008 Joint Interoperability Test Command (JTIC) Status Report", Defense Information Systems Services Agency, Washington DC, 2 March 2009.

[3] C.E. Dickerson and D. N. Mavris, "Architecture and Principles of Systems Engineering", CRC Press Auerbach Publications, Boca Raton Florida, 2009.

[4] C. Dickerson and D. Mavris, "Relational Oriented Systems Engineering (ROSE): Preliminary report," Proc. IEEE SoSE '11, Albuquerque, 2011.

[5] G. Klir, "Facets of Systems Engineering", Plenum Press, New York, 1991.

[6] International Organization of Standards (ISO), "Architecture Description", ISO/IEC Standard 42010 JTC1/SC7/WG42, 2010.

[7] J. Katz and J. Fodor, "The Structure of a Semantic Theory", Massachusetts Institute of Technology, Linguistic Society of America, Vol.88, No.1, March 2012.

[8] D. Kirk, M. Roper and M. Wood, "Identifying and Addressing problems in object-oriented framework reuse", Empirical Software Engineering, No3 Vol.12, June 2007.

[9] C. Dickerson and R. Valerdi, "Using relational model transformations to reduce complexity in SoS requirements traceability: Preliminary investigation”, Proc. IEEE SoSE '10, Loughborough, 2010.

[10] C.E. Dickerson, et al. "Using Architectures for Research, Development, and Acquisition". Defense Technical Information Center: (www.dtic.mil) AD No. ADA427961. Washington, DC, 2003.

[11] Department of Defense, "Department of Defense Architecture Framework (DoDAF), Volume I: Definitions and Guidelines, Volume II": Product Descriptions. Washington, DC, 2007.

[12] Byron Baker, "Combined Interoperability Test Plan (CITP) for Tactical Data Links (TDL) and United States Message Text Format", Defense Information Systems Agency, Washington DC, April 2007.

[13] C. Dickerson, "Mathematical Foundations for Relational Oriented Systems Engineering (ROSE)", Proc. IEEE SoSE '11, Albuquerque, 2011

[14] Department of Defense. 2008. Joint Publication 1-02 Dictionary of military and associated terms. http://www.dtic.mil/doctrine/jel/new_pubs/jp1_02.pdf.

[15] Air Land Sea Application Centre, "Introduction to Tactical Digital Information Link J and Quick Reference Guide", June 2000.

[16] A.Salo et al., "Decision Analysis: Improved Methods for Resource Allocation", New York, Springer, 2011.

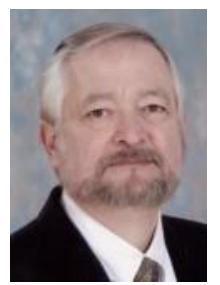

Charles Dickerson (M'06) received the Ph.D. from Purdue University, West Lafayette, IN, in 1980.

$\mathrm{He}$ is the Royal Academy of Engineering Chair of Systems Engineering at Loughborough University in the U.K. He has authored numerous papers as well as a graduate level textbook with the Georgia Institute of Technology on systems engineering and architecture. He has also co-authored an internationally recognized book on military systems architecture as well as key government reports. As a member of the IEEE, the Chair of the INCOSE Architecture Working Group, and Chair of the Mathematical Formalisms Group at the OMG, he works with the systems engineering community on systems architecture practice and standards.

Before joining Loughborough University, he was a Technical Fellow at BAE Systems, providing corporate leadership for architecture-based and system of systems engineering. He has also served as Aegis Systems Engineer for the U.S. Navy Ballistic Missile Defense Program and later as the Director of Architecture for the Chief Engineer of the U.S. Navy. Previously, as a member of MIT Lincoln Laboratory, he conducted research and tests on electromagnetic scattering. His aerospace experience includes air vehicle survivability and design at the Lockheed Skunk Works and at Northrop Advanced Systems, and operations analysis at the Center for Naval Analyses.

\section{REFERENCES}

\footnotetext{
Lexicon for Enabling Simulation (TADIL-TALES)", 10th International Command and Control Research and Technology Symposium, 2005.

[1] Joe Sorroche, "Tactical Digital Information Link-Technical Advice and
} 